\title{
Correlation Between Thrombospondin-1 Expression in Non-cancer Tissue and Gastric Carcinogenesis
}

\author{
HIDEYA KASHIHARA, MITSUO SHIMADA, KOZO YOSHIKAWA, JUN HIGASHIJIMA, \\ TAKUYA TOKUNAGA, MASAAKI NISHI, CHIE TAKASU and DAICHI ISHIKAWA
}

Department of Surgery, Institute of Health Biosciences, University of Tokushima, Tokushima, Japan

\begin{abstract}
Background/Aim: Thrombospondin-1 (TSP1) is correlated with carcinogenesis occurring in cases of intestinal inflammation. The aim of this study was to clarify the role of TSP1 in gastric carcinogenesis. Materials and Methods: A total of 39 patients with gastric cancer who had undergone gastrectomy were enrolled. The expression of TSP1 mRNA in non-cancer tissues was determined. Furthermore, the expression of CD36, STAT3 and TGF $\beta R 2$ mRNA in non-cancer tissues in two expression groups, the TSP1 high-and lowexpression groups, were examined. Results: The expression of TSP1 was high in the mucosal-atrophy group and tended to be high in the Helicobacter pylori (H. pylori) (+) and multiple cancer groups. The levels of CD36, STAT3 and TGF $\beta R 2$ mRNA were significantly higher in the TSP1-high group. TSP1 signaling pathway was induced in multiple cancer or atrophy $(+)$ or H.pylori (+) compared to cases with single cancer, atrophy (-) and H. pylori (-). Expression of proteins involved in the TSP1 signaling pathway in non-cancer tissues with multiple gastric cancers were higher than that with single gastric cancer. Conclusion: Expression of TSP1 in non-cancer tissue correlated with gastric carcinogenesis.
\end{abstract}

Gastric cancer is the third most common cause of cancer death worldwide (1). Therefore, it is very important to detect the potential molecular biomarkers for treating gastric cancer.

It is well known that the majority of gastric cancers are associated with Helicobacter pylori (H. pylori) infection (2). Chronic inflammation, such as induced $H$. pylori infection, plays an important role in the development of gastric cancer (3).

Correspondence to: Prof. Hideya Kashihara, Department of Surgery, Institute of Health Biosciences, The University of Tokushima, 3-1815 Kuramoto-cho, Tokushima, 770-8503, Japan. Tel: +81886337137 , Fax: +81 886319698, e-mail: kashihara.hideya@tokushima-u.ac.jp

Key Words: Thrombospondin-1 (TSP-1), Helicobacter pylori $(H$. pylori), transforming growth factor beta (TGF- $\beta$ ), signal transducer and activator of transcription 3 (STAT3).
Thrombospondin-1 (TSP1) is a multifunctional $450 \mathrm{kDa}$ extracellular matrix glycoprotein that is predominantly stored in platelets but also secreted at lower levels by many cell types (4). TSP1 is involved in tumor migration, invasion and the activation of the transforming growth factor (TGF-beta) (5). In addition, TSP1 might promote bacterial colonization and inflammation (6). CD36, which is a TSP1 receptor, modulates inflammation. TSP1 activates the NF-kB pathway. This pathway is critical for further activation of the signal transducer and activator of transcription 3 (STAT3). A crosscommunication between NF-kB and STAT3 induced by TSP1 plays an important role in inflammation and cancer (7). It has been previously reported that TSP-1 enhances angiogenesis due to its positive correlation with the vascular endothelial growth factor (VEGF) and microvessel density (MVD), and that it is a good prognostic factor for survival in advanced gastric cancer (8). However, the role of TSP1 in inflammation-induced gastric carcinogenesis remains unclear. The aim of this study was to investigate the role of TSP1 in gastric carcinogenesis.

\section{Materials and Methods}

A total of 39 patients undergoing gastrectomy for gastric cancer at the Tokushima University Hospital were included in this study (age range $=71.5$ years \pm 12.2 ; Male/Female: $24 / 15$ respectively; and Stage I/II/III/IV: 12/7/11/9). The regular follow-ups included monthly measurements of carcino-embryonic antigens and carbohydrate 199 levels and computed tomography and ultra-sonography every three months. Macroscopic and microscopic types, staging, and curability were defined according to the Japanese Classification of Gastric Carcinoma (the 3rd English edition, corresponds to the 14th Japanese edition) (9). Regarding tumor differentiation, tumors were divided into differentiated and undifferentiated adenocarcinomas. The $\mathrm{T}$ factor (=Tumor depth) was determined by tumor depth and was divided into two groups $(\leq \mathrm{MP}$ or $\mathrm{MP}<$ ). In terms of the $\mathrm{N}$ (=Lymph node metastasis) and P (Peritoneal dissemination) factors, they were also divided into two groups (metastasis $(+)$ or $(-)$ ). Lymphatic and venous invasion characteristics were divided into a positive or negative group. Table I shows the clinico-pathological characteristics of the patients with gastric cancer included in this 
study. The gastric mucosal inflammation and atrophy in histological findings were evaluated by pathologists based on the Sydney system (10). The pre-operative value of anti- $H$. pylori antibodies was measured in all patients. The cut-off value was $10 \mathrm{U} / \mathrm{ml}(H$. pylori $(+) /(-): 28 / 11)$.

RNA Isolation and Quantitative Real time RT-PCR. A total of 39 patients with gastric cancer were randomly selected and the biopsy samples obtained from the gastric cancer were isolated. RNA was extracted using the RNeasy mini kit (Qiagen, Valencia, CA). RNA was reverse transcribed with the high capacity cDNA reverse transcription kit (Applied Biosystems, Tokyo, Japan). Quantitative real-time RT-PCR was performed using the Applied Biosystems 7500 real-time PCR system; the TaqMan gene expression assayson-demand; and the TaqMan universal master mix (Applied Biosystems, Tokyo, Japan). The following assays (assay identification numbers) were used: TSP1 (Hs00962908_m1); CD36 (Hs01046830_m1); TGFßR2 (Hs00234253_m1); and STAT3 (Hs00374280_m1). GAPDH (4326317E) was used as a control gene. The thermal cycler conditions were as follows: two minutes at $50^{\circ} \mathrm{C}$; ten minutes at $95^{\circ} \mathrm{C}$; then forty cycles of $15^{\circ} \mathrm{C}$ at $95^{\circ} \mathrm{C}$; and one minute at $60^{\circ} \mathrm{C}$. Amplification data were analyzed with an Applied Biosystems Prism 7500 Sequence Detection System ver.1.3.1 (Applied Biosystems, Tokyo, Japan).

Statistics. For comparison of continuous variables, the MannWhitney $U$-test was used. All statistical analyses were performed using Stat View-J 5.0 software (SAS, Cary, NC). Statistical significance was defined as a $p$-value of less than 0.05 .

\section{Results}

TSP1 expression in mucosal atrophy, H. pylori and multiple gastric cancers. In the mucosal atrophy group $(n=18)$, the expression of TSPI mRNA in non-cancer tissue was significantly higher than that in the non-mucosal atrophy group $(n=21)$. In addition, the TSP1 expression in the multiple gastric cancer group $(n=12)$ tended to be higher compared with that in the single gastric cancer group $(n=27)$. However, TSP1 was not correlated with $H$. pylori infection (Figure 1).

The correlation among TSP1, CD36, TGF $\beta R 2$ and STAT3. Our hypothesis suggested that TSP1 might induce gastric carcinogenesis via the activation of CD36, TGF $\beta$ R2 and STAT3. The samples were generally classified into two groups: the TSP1 high expression group and the TSP1 low expression group according to the median value of TSP1/GAPDH. In the TSP1 high expression group, CD36, TGF $\beta$ R2 and STAT3 expressions in non-cancer tissues were significantly higher than that in TSP1 low expression group (Figure 2).

The TSP1 signaling pathway in multiple cancer or atrophy (+) or H. pylori (+). TSP1, TGF $\beta \mathrm{R} 2$ and STAT3 expressions in non-cancer tissues with multiple cancers or atrophy $(+)$ or
Table I. Clinico-pathological variables in TSP1-low and high expression groups.

\begin{tabular}{lcc}
\hline Variables & TSP1 mRNA & $p$-Value \\
\hline Age: $<70 / 70 \leq$ & $18.1 / 20.2$ & 0.57 \\
Gender: male/female & $20.8 / 17.5$ & 0.36 \\
Differentiation: & & \\
Differentiation/Undifferentiation & $20.1 / 18.9$ & 0.75 \\
Tumor depth: $\leq$ MP/MP< & $20.1 / 19.3$ & 0.86 \\
Lymph node metastasis: -/+ & $20.3 / 19.0$ & 0.72 \\
Stage I,II/III,IV & $18.9 / 20.0$ & 0.76 \\
Peritoneal dissemination: $-/+$ & $19.8 / 18.6$ & 0.77 \\
Lymphatic invasion: $-/+$ & $21.8 / 18.3$ & 0.36 \\
Venous invasion: $-/+$ & $16.7 / 21.8$ & 0.16 \\
Inflammation: $-/+$ & $12.6 / 16.4$ & 0.27 \\
\hline
\end{tabular}

The TSP 1 mRNA values correspond tothe levels of TSP1 mRNA relative to GAPDH mRNA levels.

H. pylori $(+)(\mathrm{n}=33)$ recorded significantly higher than that with single cancer and atrophy $(-)$ and $H$. pylori $(-)(\mathrm{n}=6)$ (Figure 3a-d).

The samples were generally classified into four groups according to single or multiple cancer, atrophy and $H$. pylori: none, one positive, two positives and all positives groups. In Figure 3e, TSP1 expressions increased as the number of positives increased.

TSP1 signaling pathway in multiple gastric cancers. TSP1 and TGF $\beta$ R2 in the non-cancer tissue of the multiple gastric cancer group $(n=12)$ were significantly higher than that in the single gastric cancer group $(n=27)$. STAT3 tended to be high in the multiple gastric cancer groups. CD36 expression showed no significant difference in the two groups (Figure 4).

\section{Discussion}

In this study, the TSP1 expression in non-cancer tissue compared to gastric cancer was investigated. The TSP1 expression in non-cancer tissue was high in mucosal atrophy, $H$. pylori infection and multiple cancers. CD36, STAT3 and TGF $\beta$ R2 mRNA in the TSP1 high group were significantly high. In the cases with multiple cancer, atrophy or H.pylori, expression of proteins in the TSP1 signaling pathway were increased. Therefore, the results of the study indicated that the expression of TSP1 in non-cancer tissue correlated with gastric carcinogenesis.

$H$. pylori is an etiological agent in gastrointestinal infections. The presence of H. pylori within gastric mucosa induces chronic gastritis, intestinal metaplasia, dysplasia and eventually gastric cancer (11). Chronic $H$. pylori infection within the stomach causes inflammation in the gastric 

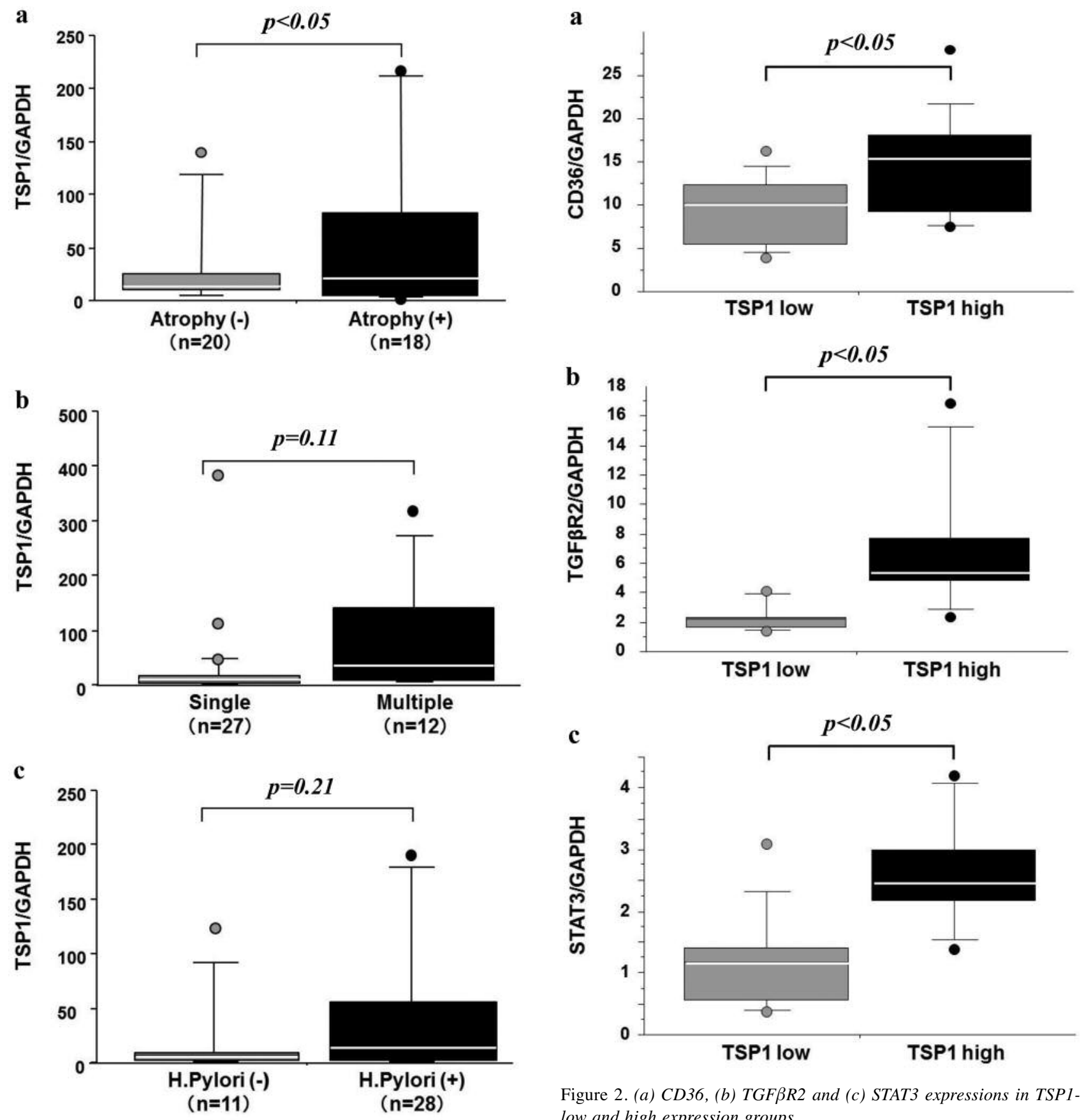

Figure 1. The expression of TSP1 in non-cancer tissue with (a) gastric mucosal atrophy (b) multiple gastric cancer and (c) H. pylori.

mucosa and can activate oncogenic pathways (12). H. pylori infection in chronic gastritis and gastric carcinoma is reported to be associated with methylation of TSP-1 (13). Therefore, this study investigated the role of TSP1 in inflammation-induced gastric carcinogenesis.

Figure 2. (a) CD36, (b) TGF $\beta R 2$ and (c) STAT3 expressions in TSP1low and high expression groups.

Some studies have emphasized the role of TSP-1 in cancer and its metastasis. TSP-1 has also key functions in inflammation and consequently in carcinogenesis through activating TGF $\beta$ (14). TSP1 has also been reported to play an important role in the colorectal adenoma to carcinoma transition (15). TSP-1 is released during the acute phase of inflammation and plays an important role in chronic 

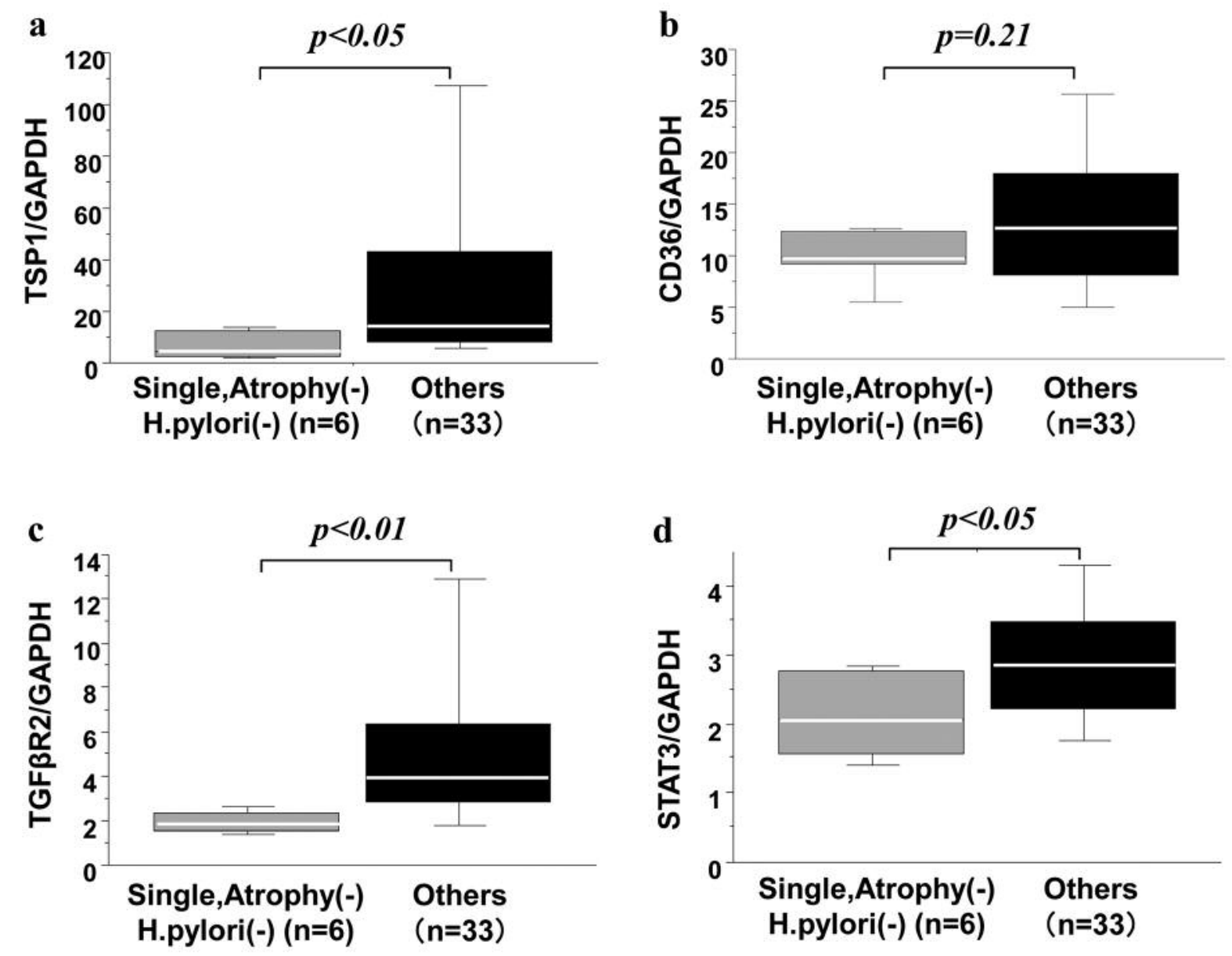

※Others; multiple cancer or atrophy (+) or H.pylori (+)

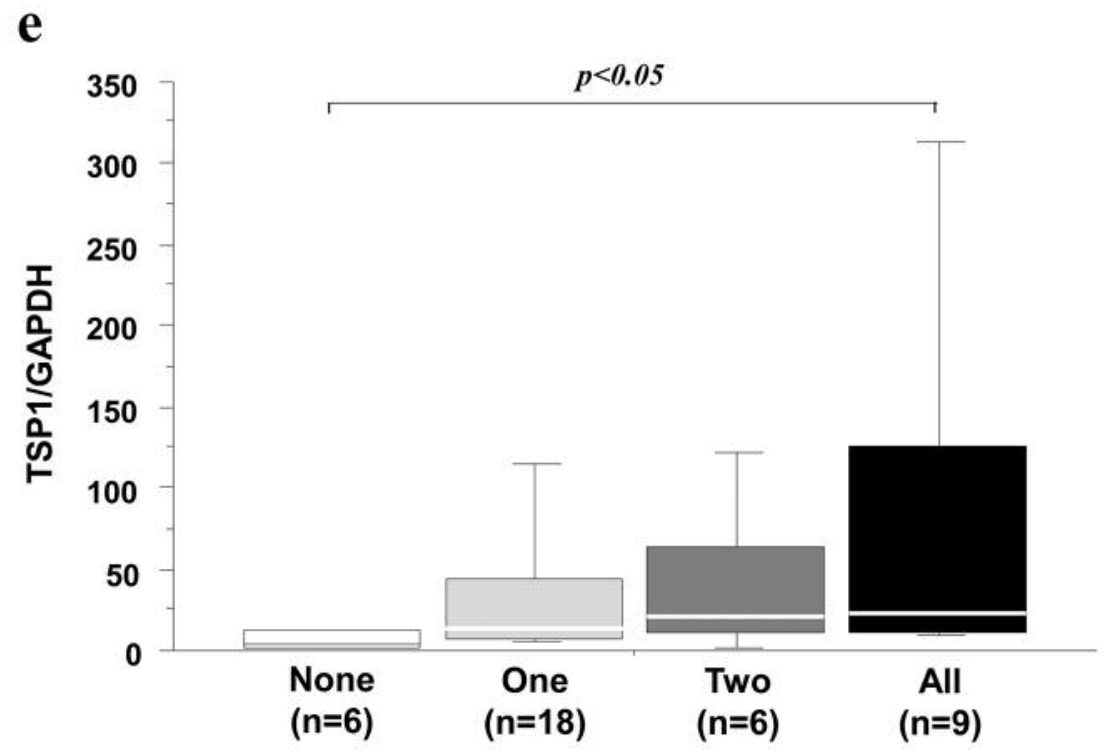

Figure 3. The comparison of (a) TSP1, (b) CD36, (c) TGFBR2 and (d) STAT3 expressions in non-cancer tissue between single cancer and atrophy $(-)$ and H. pylori (-) and multiple cancer or atrophy (+) or H. pylori (+). (e): TSP1 expressions according to the number of positives; single or multiple cancer, atrophy and H. pylori. 

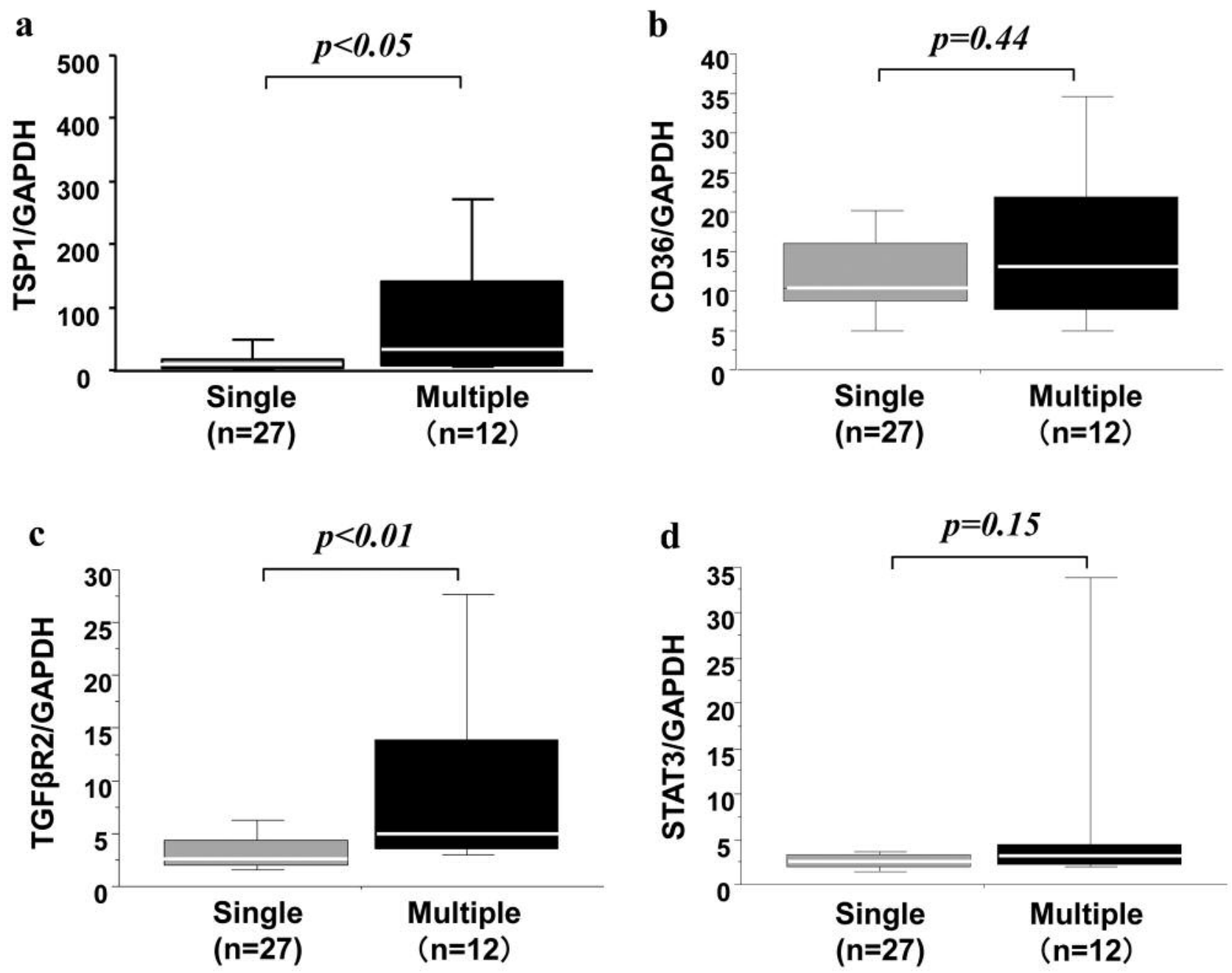

Figure 4. (a) TSP1, (b) CD36, (c) TGFßR2 and (d) STAT3 expressions in H. pylori infection with single and multiple gastric cancer.

inflammation, where TSP-1 activates the macrophage TGF $\beta$ and the NFkB pathway by binding CD36 (16). On the one hand, endothelial cells activated by TSP-1 influence the inflammatory reaction and promote carcinogenesis (14) and on the other, inflammatory bowel disease (IBD) up-regulates factors such as tumor necrosis factor-alpha (TNF $\alpha)$, TGF $\beta$ receptors 2 and 3 (TGF $\beta$ R2, 3) and interleukin 10 (IL-10) (17). Activated STAT3 is reported as a crucial factor in intestinal carcinogenesis in colitis-associated cancer (18). IL6/STAT3 signaling by latent TGF $\beta$ induces carcinogenesis upon chronic inflammation (14).

In this study, chronic inflammatory status such as multiple cancer, atrophy (+) and $H$. pylori (+) in the gastric noncancer tissues activated the TSP-1 signaling pathway. These conditions might induce gastric carcinogenesis via activation of the TSP-1 signaling pathway.
A limitation of this study was the small number of samples used. Additionally, the detailed mechanism concerning the TSP-1 signaling pathway in gastric carcinogenesis remains unclear. Consequently, further investigation on this matter will be needed.

However, this is the first report regarding the correlation between gastric carcinogenesis and the TSP-1 signaling pathway. Therefore, the detailed investigation undertaken in this study is considered to be valuable.

In conclusion, the results of this study indicate that there is a correlation between TSP1 expression in non-cancer tissues and gastric carcinogenesis. Especially, in the status of multiple cancers, mucosal atrophy and $H$. pylori infection, TSP-1 signaling pathway is activated. TSP-1 may be a possible new target for treating and preventing gastric cancer. 


\section{Conflicts of Interest}

The Authors have no conflicts of interest.

\section{References}

1 Ferlay J, Soerjomataram I, Dikshit R, Eser S, Mathers C, Rebelo M, Parkin DM, Forman D and Bray F: Cancer incidence and mortality worldwide: sources, methods and major patterns in GLOBOCAN 2012. Int J Cancer 136(5): E359-386, 2015.

2 Uemura N, Okamoto S, Yamamoto S, Matsumura N, Yamaguchi S, Yamakido M, Taniyama K, Sasaki N and Schlemper RJ: Helicobacter pylori infection and the development of gastric cancer. N Eng J Med 345(11): 784-789, 2001.

3 Chiba T, Marusawa $\mathrm{H}$ and Ushijima T: Inflammation-associated cancer development in digestive organs: mechanisms and roles for genetic and epigenetic modulation. Gastroenterology 143(3): 550-563, 2012.

4 Raugi GJ, Olerud JE and Gown AM: Thrombospondin in early human wound tissue. J Invest Dermatol 89: 551-554, 1987.

5 Roberts DD: Regulation of tumor growth and metastasis by thrombospondin-1. FASEB J 10: 1183-1191, 1996.

6 Sargiannidou I, Zhou $\mathrm{J}$ and Tuszynski GP: The role of thrombospondin-1 in tumor progression. Exp Biol Med 226(8): 726-733, 2001.

7 Eckmann L, Nebelsiek T, Fingerle AA, Dann SM, Mages J, Lang R, Robine S, Kagnoff MF, Schmid RM, Karin M, Arkan $\mathrm{MC}$ and Greten FR: Opposing functions of IKK $\beta$ during acute and chronic intestinal inflammation. Proc Natl Acad Sci USA 105(39): 15058-15063, 2008.

8 Nakao T, Kurita N, Komatsu M, Yoshikawa K, Iwata T, Utsunomiya $\mathrm{T}$ and Shimada M: Expression of thrombospondin1 and Ski are prognostic factors in advanced gastric cancer. Int J Clin Oncol 16(2): 145-152, 2011.

9 Japanese Gastric Cancer Association: Japanese Classification of Gastric Carcinoma (JCGC) (3rd English edition, corresponds to the Japanese 14th edition). Gastric Cancer 14: 101-112, 2011.

10 Dixon MF, Genta RM, Yardley JH and Correa P: Classification and grading of gastritis. The updated Sydney System. International Workshop on the Histopathology of Gastritis, Houston 1994. Am J Surg Pathol 20(10): 1161-1181, 1996.
11 Correa P: Human gastric carcinogenesis: a multistep and multifactorial process - First American Cancer Society Award Lecture on Cancer Epidemiology and Prevention. Cancer Res 52(24): 6735-6740, 1992.

12 Ding SZ, Goldberg JB and Hatakeyama M: Helicobacter pylori infection, oncogenic pathways and epigenetic mechanisms in gastric carcinogenesis. Future Oncol 6(5): 851-862, 2010.

13 Alvarez MC, Ladeira MS, Scaletsky IC, Pedrazzoli J Jr. and Ribeiro ML: Methylation pattern of THBS1, GATA-4, and HIC1 in pediatric and adult patients infected with Helicobacter pylori. Dig Dis Sci 58(10): 2850-2857, 2013.

14 Lopez-Dee Z, Pidcock K and Gutierrez LS: Thrombospondin 1: multiple paths to inflammation. Mediators Inflamm 2011: 296069, 2011.

15 Kim HC, Roh SA, Ga IH, Kim JS, Yu CS and Kim JC: CpG island methylation as an early event during adenoma progression in carcinogenesis of sporadic colorectal cancer. J Gastroenterol Hepatol 20(12): 1920-1926, 2005.

16 Leung LL, Li WX, McGregor JL, Albrecht G andHoward RJ: CD36 peptides enhance or inhibit CD36-thrombospondin binding. A two-step process of ligand-receptor interaction. J Biol Chem 267(25): 18244-18250, 1992.

17 Chidlow JH Jr, Langston W, Greer JJ, Ostanin D, Abdelbaqi M, Houghton J, Senthilkumar A, Shukla D, Mazar AP, Grisham MB and Kevil CG: Differential angiogenic regulation of experimental colitis. Am J Pathol 169(6): 2014-2030, 2006.

18 Rose-John S, Mitsuyama K, Matsumoto S, Thaiss WM and Scheller J: Interleukin-6 trans-signaling and colonic cancer associated with inflammatory bowel disease. Curr Pharm Des 15(18): 2095-2103, 2009
Received May 5, 2017

Revised May 30, 2017

Accepted May 31, 2017 